\title{
NUCLEAR PERIPHERY IN MEAN-FIELD MODELS
}

\author{
A. BARAN and P. MIERZYŃSKI \\ Institute of Physics, University of M. Curie-Sktodowska, \\ ul. Radziszewskiego 10, 20-031 Lublin, Poland \\ Received (October 12, 2003) \\ Revised (revised date)
}

\begin{abstract}
The halo factor is one of the experimental data which describes a distribution of neutrons in nuclear periphery. In the presented paper we use Skyrme-Hartree (SH) and the Relativistic Mean Field (RMF) models and we calculate the neutron excess factor $\Delta_{B}$ defined in the paper which differs slightly from halo factor $f_{\text {exp. The results of the }}$ calculations are compared to the measured data.
\end{abstract}

\section{Introduction.}

One of the important problems of nuclear physics is the description of the periphery of the nucleus. From theoretical and experimental point of view the topic is complex and much information is needed in order to predict neutron or proton excess in the neighbourhood of nuclear surface. ${ }^{1,2,3}$ The peripheral density distributions of protons and neutrons are determined theoretically by single particle eigenfunctions of protons and neutrons in this region. Applying different nuclear models one calculates wave functions in the deformed oscillator basis. They behave asymptotically like $\exp \left(-\gamma r^{2}\right)$, where $\gamma$ is a constant and $r$ is the distance from the center of the nucleus and do not reproduce properly the particle densities at large distances. The real nuclear densities behave like $\exp (-\beta r)$. In the following we rather stay in the nuclear interior where the density distributions are well defined and we describe the nuclear peripheral properties using only well defined theoretical internal nuclear densities. We would like to show that a simple function $\delta_{B}(\vec{r})$ introduced by Bethe ${ }^{5}$

$$
\delta_{B}(\vec{r})=\frac{Z}{N} \rho_{N}(\vec{r})-\rho_{Z}(\vec{r})=\tilde{\rho}_{N}(\vec{r})-\rho_{Z}(\vec{r}),
$$

allows to predict the experimentally observed halo data without the knowledge of the local behaviour of a tails of nuclear densities in asymptotic region. The function given by Eq.(1) is the difference of the relative neutron density $\tilde{\rho}_{N}(\vec{r})=(Z / N) \rho_{N}$ and the proton density $\rho_{Z}(\vec{r})$ at a point $\vec{r}$.

In the following section we introduce a neutron excess factor (NEF) based on $\delta_{B}$ defined in Eq. (1) function and we calculate NEF for a class of nuclei studied at Low Energy Antiproton Ring (LEAR) facility in CERN. ${ }^{1,2}$. Two mean field models are used in calculating NEF which after that is compared to the experimental data. 
2 A. Baran and P. Mierzyński

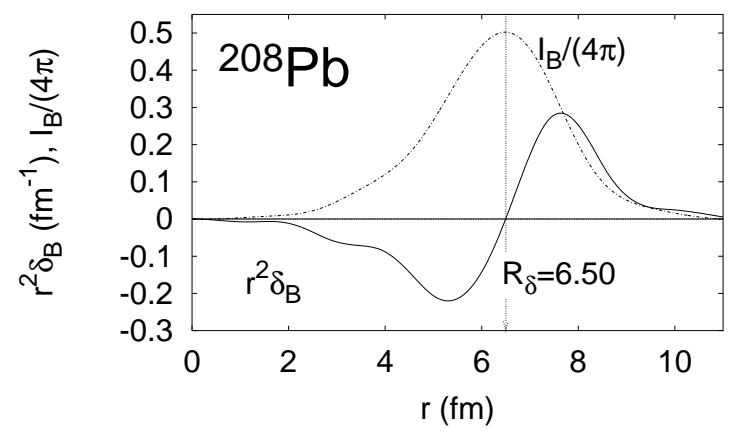

Fig. 1. Bethe function of ${ }^{208} \mathrm{~Pb}$ (multiplied by $r^{2}$, solid line) and the integral $I_{B}(r)$ (dashed line). The maximum of $I_{B}$ corresponding to $R=6.5 \mathrm{fm}$ (zero of $\delta_{B}(r)$ ) is the neutron excess factor discussed in the text.

\section{Model}

It is a trivial to notice that the integral of the Bethe function (1) over the whole space vanishes

$$
\int \delta_{B}(\vec{r}) d^{3} \vec{r}=0
$$

This direct property allows to define the total peripheral neutron or proton excess without the knowledge of the density distribution in the nuclear periphery. For example, in the case of the spherical symmetry equation (2) reads

$$
4 \pi \int_{0}^{\infty} r^{2} \delta_{B}(r) d r=4 \pi \int_{0}^{r^{\prime}} r^{2} \delta_{B}(r) d r+4 \pi \int_{r^{\prime}}^{\infty} r^{2} \delta_{B}(r) d r=0
$$

where $r^{\prime}$ is an arbitrary point which if chosen properly will maximize the first term in the above equation. Let us define the following function of the distance $r$

$$
I_{B}(r)=-4 \pi \int_{0}^{r} r^{\prime 2} d r^{\prime}
$$

The maximal value of $\Delta_{B}$ with respect to $r$

$$
\Delta_{B}=\max _{r} I_{B}(r),
$$

gives the relative outer excess of neutrons or protons out of the point which maximizes $I_{B}$. The positive value of $\Delta_{B}$ indicates the excess of neutrons while the negative value of it corresponds to the case of the relative proton excess. In the following we call the value of $\Delta_{B}$ the neutron excess factor (NEF). Both $\delta_{B}$ and $B$ functions for ${ }^{208} \mathrm{~Pb}$ are shown in Figure 1.

Similar functions and quantities are defined for more general case of a deformed nuclei. ${ }^{4}$ The corresponding integrals and quantities (Eqs. 4 and 5) are calculated in this case by taking the integrals over the region of the deformed surface similar to the average nuclear surface. 


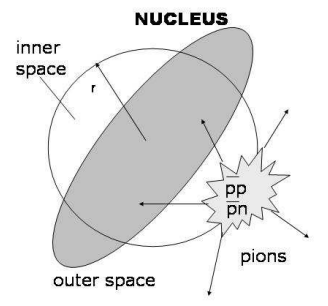

Fig. 2. Annihilation of $\bar{p} p$ or $\bar{p} n$ on the periphery of the deformed nucleus. The inner and the outer regions are divided by a sphere of radius $r$. The pions are the outcome of the annihilation reaction.

\section{Antiproton annihilation on nucleus}

The measurements of products of the annihilation of antiprotons on nuclei ${ }^{1,2}$ inform on the value of the neutron excess in the nuclear periphery where the annihilation takes place. This is the outer region extending over the whole periphery of the nucleus. From the experiment ${ }^{1,2}$ one approximatelly knows the average radius of stopping of antiprotons. It is slightly larger (by about $2 \mathrm{fm}$ ) than the average nuclear radius $R=r_{0} A^{1 / 3}$.

While the deformed nuclei are not oriented in the target the information about the process is contained in the averages which depend neither on the direction nor on the distance from the center of the nucleus (see Fig. 2). This corresponds to the averaging over the outer region. Assuming the annihilation cross section locally proportional to the density of nucleons (protons or neutrons) one sees that the ratio of the cross section of stopping the antiproton on neutrons to the cross section of stopping antiproton on protons is proportional to corresponding ratio of the densities $\rho_{N} / \rho_{Z}$. The total annihilation ratio is thus approximately proportional to the integral of the ratio of the densities over the whole outer region. A similar averaging has been performed in calculations of $\Delta_{B}$ and therefore the result given in Eq. (5) i.e., NEF is proportional to the halo factor $f_{\exp }$ (see Eq. (6)) defined by ${ }^{1,2}$

$$
f_{\exp } \sim \frac{Z}{N} \frac{\mathcal{N}_{\bar{p} n}}{\mathcal{N}_{\bar{p} p}},
$$

where $\mathcal{N}_{\bar{p} p}$ and $\mathcal{N}_{\bar{p} n}$ denote the number of annihilations of both $\bar{p} p$ and $\bar{p} n$ types respectively (the final number of $Z_{-1} X^{N}$ nuclei corresponding to $\bar{p} p$ annihilation and the final number of ${ }_{Z} X^{N-1}$ nuclei which correspond to $\bar{p} n$ annihilation of $\bar{p}$ on a nucleus ${ }_{Z} X^{N}$ ) The final nuclei are "counted" after the anihilation processes has taken place.

\section{Results}

In the following section we compare our results of calculations of $\Delta_{B}$ to the experimentally measured halo factor $f_{\text {exp }}$. 


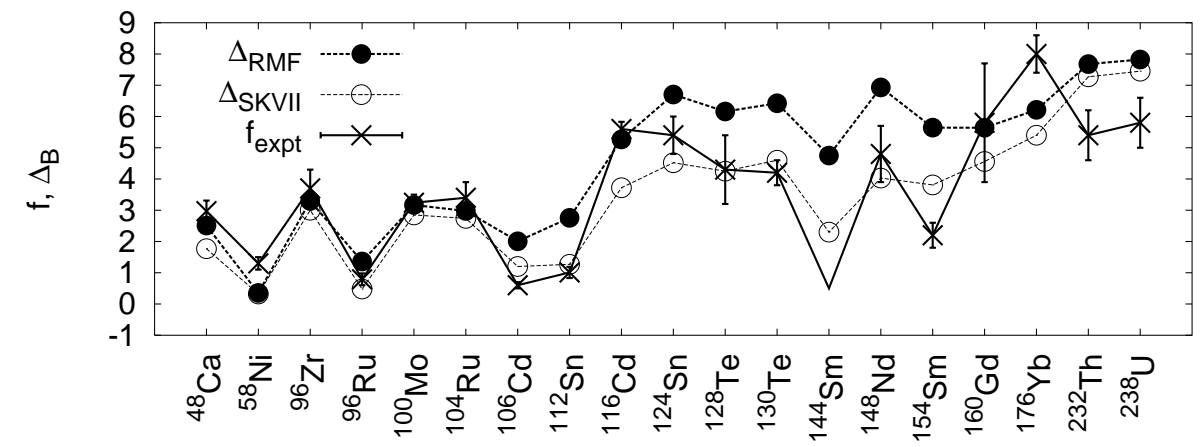

Fig. 3. Neutron excess factor $\Delta_{B}$ (NEF) for nuclei studied by the Low Energy Antiprotonic Ring (LEAR) group at CERN. Experimental data are given with crosses connected by solid line. Plain circles represent the RMF data and the open circles (dashed line) correspond to Skyrme-Hartree calculations with SkVII force. For more details and the references see the text.

Figure 3 shows the outer neutron excess factor $\left(\Delta_{B}\right)$ as calculated in the Relativistic Mean Field model (RMF, plain circles) with NL3 set of parameters ${ }^{6}$ and in the selfconsistent Skyrme-Hartree (SH) model with SkVII force (open circles). ${ }^{7}$ The data are presented for nuclei reported by Low Energy Antiprotonic Ring group (LEAR) at CERN. ${ }^{1,2}$ It is observed that the calculations in both RMF and SH models are strongly correlated with experimental halo factor $f_{\exp }$ (the scale of $\Delta_{B}$ is chosen arbitrarilly). The correlation shows that the NEF $\Delta_{B}$ is a good indicator of the neutron halo property and it gives the measure of the peripheral annihilation ratio of the antiproton on the neutrons and protons in the nuclear system.

\section{References}

1. P. Lubiński, J. Jastrzȩbski, A. Trzcińska, W. Kurcewicz, F.J. Hartmann, R. Schmidt, T. von Egidy, R. Smolańczuk, and S. Wycech, Phys. Rev. C 57, 2962, (1998)

2. R. Schmidt, F. J. Harrtmann, B. Ketzer, T. von Egidy, T. Czosnyka, J. Jastrzȩbski, M. Kisieliński, P. Lubiński, L. Pieńkowski, A. Trzcińska, B. Kłos, R. Smolańczuk, S. Wycech, W. Pöschl, K. Gulda, W. Kurcewicz, and E. Widmann. Phys. Rev. C 60, 054309, (1999)

3. A. Baran, K. Pomorski, and M. Warda, Z. Phys A 357, 33, (1997).

4. A. Baran, P. Mierzyski, submitted to Acta Physica Polonica B.

5. H. A. Bethe, Ann. Rev. Nucl. Sci., 21, 93, (1970).

6. G. Lalazissis, J. König, , and P. Ring. Phys. Rev. C 55, 540, (1996).

7. M. J. Giannoni, P. Quentin, Phys. Rev., 21, 2076, (1980). 